\title{
AVALIAÇÃO DOS EFEITOS DO TREINAMENTO NO PERÍODO PREPARATÓRIO EM ATLETAS PROFISSIONAIS DE FUTEBOL
}

\author{
DR. JOÃO PAULO BORIN \\ Doutor em Educação Física pela UNICAMP e Professor da Faculdade \\ de Educação Física da Universidade Estadual de Campinas - FEF (São Paulo - Brasil) \\ e-mail: borinjp@fef.unicamp.br \\ GRAD. RICARDO STOCHI DE OLIVEIRA \\ Licenciado em Educação Física pela UNIMEP (São Paulo - Brasil) \\ e-mail: borinjp@fef.unicamp.br \\ GRAD. MAYKEL GUILHERME DE CAMPOS \\ Licenciado em Educação Física pela UNIMEP (São Paulo - Brasil) \\ e-mail: borinjp@fef.unicamp.br

\section{GRAD. CLÁUDIO ROBERTO CREATTO Licenciado em Educação Física pela UNIMEP e Membro da Comissão Técnica SEV/Hortolandia (São Paulo - Brasil) e-mail: borinjp@fef.unicamp.br}

\section{DR. CARLOS ROBERTO PEREIRA PADOVANI}

Doutor em Agronomia pela Universidade Estadual Paulista Júlio de Mesquita Filho e

Professor do Centro Estadual de Educação Tecnológica Paula Souza, Universidade Nove de Julho e Faculdade Marechal Rondom (São Paulo - Brasil). e-mail: bioestatistica@ibb.unesp.br

\section{DR. CARLOS ROBERTO PADOVANI}

Doutor em Estatística e Experimentação Agronômica pela Universidade de São Paulo e Professor Titular do Instituto de Biociências,

Departamento de Bioestatística da Universidade Estadual

Paulista Júlio de Mesquita Filho (São Paulo - Brasil) e-mail: bioestatistica@ibb.unesp.br 


\section{RESUMO}

O objetivo do presente estudo foi avaliar os efeitos do treinamento no período preparatório em atletas profissionais de futebol. Participam 17 atletas, com idade acima de 18 anos que disputaram a terceira divisão do campeonato paulista, em 2009. O programa de treinamento predominante foi o tipo neuromuscular e por meio dos testes de sentar e alcançar, salto sêxtuplo, salto vertical e velocidade de deslocamento em 30m os atletas foram avaliados antes do início do programa de treinamento (MI) e após sete semanas (M2). Os dados coletados foram armazenados em banco computacional, produzindo-se informações no plano descritivo (medidas de centralidade e dispersão) e, no inferencial, Teste t de Student e Anova One-way. Os principais resultados apontam para melhora da flexibilidade, força explosiva e rápida, e sensível diminuição na velocidade de deslocamento em 30 metros.

PALAVRAS CHAVE: Futebol; preparação; treinamento desportivo; metodologia.

\section{INTRODUÇÃO}

Em desportos coletivos, é de grande importância obter indicadores que apontem para as necessidades, limitações e evoluções durante um processo de treinamento. Nesse sentido a avaliação do desempenho de jogadores de futebol em testes específicos, atua como ferramenta indispensável para membros da comissão técnica no conhecimento de tais indicadores e posterior prescrição do treinamento.

Particularmente no futebol, caracterizado por modalidade esportiva com ações cíclicas e acíclicas, exigindo predominantemente energia pela via aeróbia, porém nos movimentos decisivos a solicitação é anaeróbia (STOLEN et al., 2005), requer do futebolista o desempenho ótimo de capacidades biomotoras como resistência aeróbia, potência anaeróbia, velocidade de deslocamento, agilidade e força explosiva (CASTAGNA et al., 2006; BLOOMFIELD et al., 2007; DI SALVO et al., 2007; COMETTI et al., 200 I; HOFF, 2005).

Nesse sentido, destaca-se que a estruturação e desenvolvimento do treinamento destas capacidades dependerão de diferentes fatores como metodologias e conteúdo de trabalho executadas durante a temporada competitiva ou modelo de periodização adotado, dinâmica das alterações das capacidades biomotoras, sequência de cargas de treino, efeitos, adaptações e respostas aos estímulos empregados (SVENSSON, DRUST, 2005; TASKIN, 2008).

Apesar de alguns estudos apresentarem informações sobre o programa de treinamento adotado e os resultados quanto às capacidades biomotoras (BOGDANIS et al., 2007; BRAVO et al., 2008; HILL-HAAS et al., 2009; VENTURELLI; TRENTIN; BUCCI, 2007; KOTZAMANIDIS et al., 2005; MCMILLAN et al., 2005; GOROSTIAGA et al., 2004; SAMPAIO et al., 2007), verifica-se no âmbito nacional a orientação 
do treinamento baseada principalmente nas experiências práticas de treinadores, principalmente pelo método de tentativa e erro, diminuindo a possibilidade de alcance do desempenho ótimo entre as capacidades exigidas na modalidade. Há necessidade de sistematizar as metodologias de preparação para futebolistas, tendo em vista procedimentos que indiquem a longo prazo, o contínuo aumento do rendimento, levando em consideração a especificidade da modalidade bem como o nível dos resultados que se pretende alcançar.

Neste sentido a justificativa do presente trabalho volta-se em discutir a problemática apresentada no cotidiano do treinamento de uma equipe de futebol que conta durante o período preparatório com escasso tempo necessário para realização de preparação adequada. De fato, Gomes e Souza (2008) relatam que uma equipe de futebol pode realizar de 80 a 90 partidas oficiais durante a temporada competitiva e a preparação dos atletas é fundamental neste processo.

Assim o objetivo do presente trabalho é avaliar os efeitos de treinamento no período preparatório em atletas profissionais de futebol.

\section{MATERIAL E MÉTODO}

Foram estudados 17 atletas futebolistas profissionais, da cidade de Hortolândia/São Paulo, com idade acima de 18 anos, massa corporal de 72,9 $\pm 7,9 \mathrm{~kg}$, estatura de I,77 \pm 0,07 m e que não apresentaram evidências clínicas de alterações cardíacas, pulmonares e ortopédicas.

Quanto às questões éticas, após serem esclarecidas as finalidades da pesquisa e os procedimentos aos quais os futebolistas seriam submetidos, todos assinaram termo de consentimento livre e esclarecido aprovado pelo Comitê de Ética em Pesquisa da Universidade Metodista de Piracicaba - Piracicaba - Brasil, protocolo n²8/07.

Durante sete semanas, período chamado de preparatório para participação no Campeonato Paulista da série A3 (terceira divisão), em 2009, os atletas treinavam seis vezes por semana, com duração média de 90 minutos, iniciando os trabalhos com exercícios de alongamento e aquecimento muscular geral e a seguir, era executada a sessão de treinamento programada pela comissão técnica. $\bigcirc$ volume total de trabalho no período estudado foi de 3780 minutos, em 74 sessões. Quanto ao conteúdo das sessões de treinamento foi classificado de acordo com a proposta de Gomes e Souza (2008), que dividem o tipo de treinamento em funcional e neuromuscular.

Quanto ao funcional compreende as capacidades de resistência aeróbia, resistência de velocidade e especial, esta última caracterizada por treinamentos técnicos em grupo que envolva componentes da modalidade e considerem a 
posição de atuação do atleta, com atividades como cruzamentos para finalizações, exercícios específicos para sistema defensivo bem como orientações táticas, em que trabalham diferentes movimentos que podem ocorrer na competição e, dessa forma, solicitam manifestações da resistência especifica do futebolista, em situações como marcação por setor ou individual, treinamentos em campos com dimensões reduzidas e número variado de jogadores, treinamentos coletivos com ou sem limitação de toques na bola além de jogos-treino e amistosos.

No tipo neuromuscular, constam as capacidades de velocidade, coordenação, força máxima e especial, esta última entendida como a força que o atleta aplica quando realiza o gesto específico de competição (FORTEZA DE LA ROSA; FARTO, 2007), por meio de treinamentos com pesos, realizados no campo de jogo, executando movimentos que tenham maior relação com as ações motoras do jogo.

A tabela I apresenta o tipo de treinamento e respectivo valor, em minutos, do total das sessões, bem como as capacidades biomotoras pertencentes a cada tipo de treinamento e o percentual desenvolvido em cada semana estudada.

Tabela I. Distribuição percentual das capacidades biomotoras segundo semanas estudadas.

\begin{tabular}{|c|c|c|c|c|c|c|c|c|}
\hline \multirow{2}{*}{$\begin{array}{l}\text { Tipo de } \\
\text { treinamento }\end{array}$} & \multirow{2}{*}{$\begin{array}{l}\text { Capacidades } \\
\text { biomotoras }\end{array}$} & \multicolumn{7}{|c|}{ Semanas } \\
\hline & & I & 2 & 3 & 4 & 5 & 6 & 7 \\
\hline \multirow{3}{*}{$\begin{array}{l}\text { Funcional } \\
\text { (1680 minutos) }\end{array}$} & $\begin{array}{l}\text { Resistência } \\
\text { aeróbia }\end{array}$ & $60 \%$ & $40 \%$ & $30 \%$ & $20 \%$ & $20 \%$ & $20 \%$ & $20 \%$ \\
\hline & $\begin{array}{l}\text { Resistência } \\
\text { especial }\end{array}$ & $30 \%$ & $20 \%$ & $40 \%$ & $50 \%$ & $50 \%$ & $40 \%$ & $60 \%$ \\
\hline & $\begin{array}{l}\text { Resistência de } \\
\text { velocidade }\end{array}$ & $10 \%$ & $40 \%$ & $30 \%$ & $30 \%$ & $30 \%$ & $40 \%$ & $20 \%$ \\
\hline \multirow{4}{*}{$\begin{array}{l}\text { Neuromuscular } \\
\text { ( } 2100 \text { minutos) }\end{array}$} & Força máxima & $10 \%$ & $10 \%$ & $10 \%$ & $20 \%$ & $25 \%$ & $30 \%$ & $30 \%$ \\
\hline & Força especial & $30 \%$ & $30 \%$ & $30 \%$ & $20 \%$ & $20 \%$ & $20 \%$ & $25 \%$ \\
\hline & Velocidade & $50 \%$ & $50 \%$ & $50 \%$ & $55 \%$ & $55 \%$ & $45 \%$ & $40 \%$ \\
\hline & Coordenação & $10 \%$ & $10 \%$ & $10 \%$ & $5 \%$ & $5 \%$ & $5 \%$ & $5 \%$ \\
\hline
\end{tabular}

Todos os voluntários foram submetidos a uma avaliação clínica (anamnese e exame físico) antes do início dos treinamentos e a seguir, submetidos a protocolo de testes neuromusculares em dois momentos: MI : início dos treinamentos e, M2: após sete semanas, caracterizado como término do período preparatório.

Quanto aos indicadores neuromusculares foram realizados testes de força rápida e explosiva, flexibilidade e velocidade de deslocamento em $30 \mathrm{~m}$, sugeridos para equipes de futebol (BRAZ; SPIGOLON; BORIN, 2009). Em relação à força 
rápida, foi medida por meio do teste de salto horizontal sêxtuplo. $\bigcirc$ atleta parado, em pé com as pernas em afastamento ântero-posterior, com a ponta de um dos pés atrás da linha limite, realiza seis saltos consecutivos com as pernas alternadas (d/e/d/e/d/e) e, a seguir mede-se a distância do início (marca determinada) até a última passada. Os valores são expressos em metros e os atletas realizaram três saltos, sendo considerada a melhor marca (SOUZA, 2006).

Para avaliação da força explosiva de membros inferiores utilizou-se a técnica de Counter Movement Jump (CMJ) com auxílio dos braços, conforme protocolo proposto por Bosco (2007), em que o atleta fica em pé com o tronco ereto e joelhos em extensão a $180^{\circ}$, realizando o salto vertical com o contra movimento. A flexão do joelho acontece aproximadamente no ângulo de $120^{\circ}$, em seguida o executante faz a extensão do joelho, procurando impulsionar o corpo para o alto e na vertical. Os joelhos devem permanecer em extensão durante a fase de voo e o intervalo entre tentativas é de dez segundos. Três tentativas foram realizadas, sendo considerada a melhor marca e, para análise dos saltos, foi utilizada plataforma de contato CEFISE ${ }^{\circledR}$, conectada ao sistema para medida de salto Jump System ${ }^{\circledR}$.

Para mensuração da flexibilidade do quadril utilizou-se o método linear com o teste de sentar-e-alcançar, proposto originalmente por Wells e Dillon, em 1952. Neste caso seguiu-se a padronização proposta por Marins e Giannichi ( 1996), onde se utiliza o banco de Wells e o indivíduo é posicionado sentado com as pernas completamente estendidas e os pés descalços ligeiramente afastados e completamente apoiados contra o banco. Pede-se, então, ao indivíduo para realizar três tentativas de flexão do tronco, mantendo os joelhos, cotovelos e punhos em extensão procurando a posição máxima alcançada com a ponta dos dedos, para que possa ser feita a leitura na régua. A medida é anotada em centímetros.

Por fim a velocidade de deslocamento em 30 metros seguiu protocolo descrito por Svensson, Drust (2005), em que o atleta deve partir em pé junto à linha de largada e percorrer a distância prevista na maior velocidade possível. Cada futebolista realizou duas tentativas com intervalo de 5 minutos entre as tentativas, anotando-se o melhor resultado obtido $(\mathrm{m} / \mathrm{s})$. Para a mensuração do tempo nos testes utilizou-se o sistema de fotocélulas Speed Test 6.0 CEFISE ${ }^{\circledR}$.

Os dados foram armazenados em banco computacional e a seguir produziram-se informações no plano descritivo, por meio de medidas de centralidade e dispersão e, no plano inferencial, após assumir a normalidade dos dados pelo teste de Shapiro-Wilk, utilizou-se o teste t de Student pareado e Anova One-way, para verificar diferença entre os momentos do estudo, adotando-se $p<0,05$, como nível de significância (PADOVANI, 200I). 


\section{RESULTADOS}

A partir dos dados coletados os resultados dos valores neuromusculares são apresentados na tabela 2 quanto à média e desvio-padrão, segundo momento da avaliação.

Particularmente quanto à flexibilidade verificam-se valores significantemente diferentes $(\mathrm{P}<0,05)$ de $\mathrm{MI}$ para $\mathrm{M} 2$, apontando que os atletas apresentaram meIhoria nesta capacidade.

Ao buscar conhecer o deslocamento horizontal dos atletas observa-se no salto horizontal melhora significante $(P<0,05)$ entre o primeiro momento avaliado e o segundo. Tal comportamento não é verificado em relação ao salto sêxtuplo que apresenta pequeno decréscimo entre $\mathrm{MI}$ e $\mathrm{M} 2$ e, os valores médios da velocidade na distância de $30 \mathrm{~m}$ apresentaram sensível alteração no período estudado.

Tabela 2. Medidas descritivas das variáveis neuromusculares segundo momento da avaliação.

\begin{tabular}{llll}
\hline & \multicolumn{2}{l}{ Momento da Avaliação } & Resultado estatístico \\
\cline { 2 - 4 } Capacidade biomotora & $\mathrm{M}$ M2 & \\
\hline Flexibilidade & $38,6 \pm 4,7 \mathrm{~cm}$ & $43,0 \pm 3,9 \mathrm{~cm}$ & $\mathrm{P}<0,05$ \\
\hline Força explosiva & $44,0 \pm 2,15 \mathrm{~m}$ & $48,8 \pm 2,26 \mathrm{~m}$ & $\mathrm{P}<0,05$ \\
\hline Força rápida & $15,73 \pm 1,06 \mathrm{~m}$ & $15,65 \pm 0,85 \mathrm{~m}$ & $\mathrm{P}>0,05$ \\
\hline Velocidade deslocamento & $7,11 \pm 0,16 \mathrm{~m} / \mathrm{s}$ & $7,08 \pm 0,12 \mathrm{~m} / \mathrm{s}$ & $\mathrm{P}>0,05$ \\
\hline
\end{tabular}

\section{DISCUSSÃO}

Diante das situações peculiares encontradas em equipes de futebol como tempo disponível para preparação e consequentemente, decisões a serem tomadas na organização do treinamento, faz-se necessário encontrar formas de estruturação que direcionem estes desportistas ao desenvolvimento elou manutenção do conjunto das capacidades biomotoras no período competitivo, não necessariamente em níveis máximos (STOLEN et al., 2005), pois a modalidade não o exige (HOFF; HELGERUD, 2004). A partir disto, espera-se a manutenção destes níveis alcançados durante toda a temporada competitiva, especificamente das capacidades neuromusculares. Tal fato parece estar relacionado ao entendimento da distribuição do programa de treinamento voltados especificamente ao desenvolvimento da força explosiva e velocidade em futebolistas (GOMES; SOUZA, 2008).

Neste contexto, a partir dos resultados aqui obtidos parece oportuno discutir o comportamento das capacidades biomotoras frente ao programa de treinamento utilizado, em que se observa predomínio do tipo de treinamento neuromuscular, particularmente quanto à velocidade e força especial com os maiores percentuais, 
nas diferentes semanas, e no funcional, destaque para resistência tanto especial quanto de velocidade.

Quanto à flexibilidade, considerada uma variável fundamental no futebol pela relação positiva que possui quanto à execução dos gestos técnicos (WEINECK, 2000), parece que sua melhoria significativa, observada no presente trabalho, possui relação tanto com o trabalho de força como os exercícios de alongamento realizados no início das sessões além da coordenação, apesar de menor parcela percentual na distribuição do conteúdo de treinamento. Em relação à força, Fleck e Kraemer ( 1999) relatam que os exercícios com pesos estimulam os limites da amplitude das articulações, o que associado ao aumento de tecido conjuntivo, explica os efeitos benéficos sobre a flexibilidade.

Baseado nesta concepção o treinamento de força pode auxiliar no ganho de flexibilidade, e segundo Thrash e Kelly (2007), um programa de treinamento com pesos para desenvolver força muscular não prejudica a flexibilidade e pode até aumentar a amplitude de determinados movimentos. Nesta linha Todd (2005) afirma que pouca evidência científica ou empírica existe a favor da crença de que o treinamento de força resulta em diminuição da flexibilidade. Beedle et. al. (200l) constataram que levantadores olímpicos de peso tinham flexibilidade acima da média na maioria das articulações e que comparados a outros atletas ficavam inferior apenas aos ginastas. Particularmente quanto ao futebol, Pontes et al. (2005), ao estudarem 20 futebolistas profissionais com uma frequência de treinamento de força de no mínimo três vezes por semana, também relatam melhoria nos resultados do início dos trabalhos $(30,1 \pm 6,8 \mathrm{~cm})$ e após 16 semanas $(33,0 \pm 7,1 \mathrm{~cm})$. Verkhoshanski (2000) destaca que, o alongamento realizado antes e após as sessões de treinamento pode ocasionar aumento no delineamento muscular e consequentemente melhora na amplitude do movimento, podendo ainda diminuir a chance de lesões e remoção de alguns resíduos metabólicos produzidos pela sessão de treinamento.

Quanto à força explosiva, ao observar a modalidade, nota-se que o atleta realiza ações intensas e de curta duração, exigindo rápida mudança de direção, execução de saltos, dribles, desarmes, e finalizações e a capacidade de produzir força em períodos curtos de tempo está muito ligada ao desempenho desse atleta. Ao entender essa capacidade biomotora, sabe-se que alguns fatores interferem no ótimo desempenho desta, entre os quais, o tipo de movimento realizado, as condições momentâneas das estruturas morfológicas dos músculos participantes da ação, das características neurogênicas, do grau de treinamento do indivíduo, das condições hormonais que são apresentadas no momento e de modo especial, da composição muscular e o percentual de fibras rápidas (BOSCO, 2007). Além de todos esses fatores, um fator situa-se como principal que é a condição em que o 
músculo se encontra anteriormente a realização do movimento, seja em repouso, em pré-alongamento ou estático.

Ao observar os resultados dos testes observa-se aumento no salto vertical e uma ligeira diminuição nos valores do salto sêxtuplo. Quanto aos valores encontrados no salto vertical foram superiores aos encontrados por Reilly et al. (2000) e Taskin (2008). No salto sêxtuplo também os valores do presente estudo são maiores que os apresentados por Toledo (2000) e Souza (2006).

A melhoria na força explosiva pode ter ocorrido pelo fato da maior porcentagem do treinamento, no final do período, estar voltada para a capacidade de força máxima e, possivelmente o não aumento da força rápida, pode ser explicada pelo maior grau de dificuldade de sua execução do salto sêxtuplo.

De fato, atualmente no contexto do treinamento de força especial para o futebolista, o que se utiliza em uma sessão voltada ao aprimoramento desta capacidade, consiste na execução de exercícios de força máxima (alta intensidade e poucas repetições), seguidos de exercícios de força explosiva ou velocidade (sprints, pliometria, corrida tracionada, entre outras) e ainda exercícios voltados para a resistência de força, como séries com alto volume de saltos e jogos em campos com dimensões reduzidas (GOMES; SOUZA, 2008). Wisloff et al. (2004) demonstraram fortes correlações da força máxima com a força explosiva $(r=0,78)$ e velocidade de deslocamento em I0m ( $r=0,94)$ e $30 \mathrm{~m}(r=0,7 \mathrm{I})$, para futebolistas. A estimulação combinada destas capacidades atua positivamente no desenvolvimento das mesmas, como já foi previamente demonstrado por outras pesquisas (GOROSTIAGA et al., 2004; BOGDANIS et al., 2007; VENTURELLI; TRENTIN; BUCCI, 2007; NUÑEZ et al., 2008).

Entretanto, o treinamento de força máxima durante longo período da preparação também pode provocar adaptações negativas na velocidade. Acerca dos efeitos do treinamento desta capacidade biomotora, Bosco (2007) entende que se de um lado a melhora da estrutura morfológica das fibras rápidas pode representar adaptação biológica ao estímulo do treinamento, por outro, a melhora concomitante das fibras lentas e o alargamento de sua área provocariam um efeito de desaceleração durante a contração rápida do músculo, capaz de anular a contribuição positiva das fibras rápidas.

Porém deve-se também considerar que uma parcela importante da resistência especial foi prescrita no programa de treinamento e que esta capacidade possui importância fundamental para a atuação do futebolista, devendo ser considerada um componente relevante na preparação física desses atletas (GOMES; SOUZA, 2008).

Considerando tal fato, o treinamento da resistência especial deve corresponder às particularidades da modalidade em questão e o seu desenvolvimento, 
deve acontecer por meio de exercícios preparatórios especiais que apresentem grande semelhança com as formas, peculiaridades e estruturas dos exercícios competitivos.

Em investigação feita por Ferrari Bravo et al. (2007) em que aplicaram estímulos baseados no método de sprint da corrida intervalada, realizado duas vezes por semana durante sete semanas objetivando verificar a influência que este exercia sobre alguns componentes do desempenho dos futebolistas, observaram que foi eficiente para elevar os níveis de capacidade e potência aeróbia, na manutenção da altura e potência do salto com contra movimento.

De fato, poucas são as pesquisas científicas que estão avaliando a influência e efetividade dos métodos de sprints repetidos para o aperfeiçoamento das capacidades biomotoras do futebolista, de modo particular as que se atrelam estreitamente com os componentes específicos do desempenho para a modalidade.

Contrariamente, os estudos que ao redor dos jogos em campos com dimensões reduzidas têm se elevado nos últimos anos (IMPELLIZZERI et al., 2006; FERRARI BRAVO et al., 2007; LITTLE; WILLIANS, 2007; JONES; BARRY, 2007; KELLY; DRUST, 2008) e geralmente investigam a efetividade deste, como um meio para manter e elevar níveis das capacidades biomotoras, como a resistência aeróbia, anaeróbia e especial, que segundo Rampinini et al. (2007) é um meio interessante por combinar estímulos para o desenvolvimento técnico, tático e fisiológico do atleta.

Por fim quanto à velocidade de deslocamento, considerada capacidade dependente principalmente do sistema neuromuscular (CRONIN; HANSEN, 2006) e que possui como característica o recrutamento de unidades motoras fásicas características das fibras de contração rápida e velocidade de disparos dos impulsos nervosos na musculatura, além da estrutura coordenativa do movimento exigir alta velocidade de execução (BOSCO, 2007), apresenta sensível decréscimo na distância de $30 \mathrm{~m}$, entre os momentos avaliados, o que pode ser explicado inicialmente pelas adaptações ocasionadas pelo maior volume no treinamento do tipo funcional, especialmente para a resistência aeróbia, nas semanas iniciais.

Alguns estudos, como de Hennessy e Watson (1994), Gorostiaga et al. (1999), Bell et al. (2000), Núñez et al.(2008), relatam que o treinamento da resistência pode causar efeitos concorrentes no desempenho da velocidade, principalmente por serem diferentes quanto aos aspectos coordenativos dos gestos motores (KOTZAMANIDIS et al., 2005); recrutamento de unidades motoras (BOSCO, 2007); a frequência de emissão de impulsos nervosos (LEVERITT et al., 1999) e, por fim as adaptações hormonais, morfológicas (KRAEMER et al., 1995) e metabólicas (BELL et al., 2000). 
Desta forma, especula-se que maior volume de meios de treinamento que enfatizem baixa freqüência de disparos da musculatura e maior recrutamento das unidades motoras tônicas em relação às fásicas ocasione adaptações concorrentes a capacidade de velocidade máxima dos futebolistas.

Gorostiaga et al. (2004) verificaram que a corrida contínua de baixa intensidade realizada nas partidas e em meios competitivos de treinamento pode causar efeito negativo no desempenho das capacidades neuromusculares para futebolistas. De

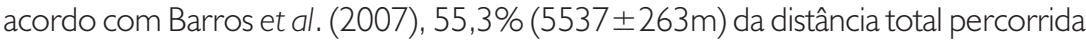
em jogos ( $10012 \pm 1024 \mathrm{~m})$ por futebolistas profissionais brasileiros ocorrem em baixa intensidade de deslocamento ( 0 a I I km/h), o que talvez justifique a prescrição do trabalho aeróbio nas semanas iniciais de preparação.

Tais informações apontam para importância da organização racional das cargas de trabalho para futebolistas, tendo como objetivo durante a sequência da temporada, a manutenção e desenvolvimento das capacidades neuromusculares, consideradas determinantes para o desempenho na modalidade. O trabalho neuromuscular deve ser prioritário dentro do plano organizacional para o desenvolvimento da preparação física especial do futebolista, seguido da estimulação metabólica específica e aprimoramento da velocidade.

\section{CONCLUSÃO}

Conclui-se que o programa de treinamento adotado no período de sete semanas ocasionou melhora na flexibilidade, força explosiva e rápida, e sensível diminuição na velocidade de deslocamento em 30m.

\section{Evaluation of training effects in preparatory period in professional soccer players.}

ABSTRACT: The purpose of this study was to evaluate the effects of training in the preparatory period in professional soccer players. Seventeen athletes, aged above 18 years were included in the study and who dispute the third division of Championship "Paulista", in 2009. The training program was the predominant type neuromuscular and through tests of sit and reach, sextuple jump, vertical jump and speed of movement in the $30 \mathrm{~m}$ athletes were evaluated before the training program (MI) and after seven weeks (M2). The data collected data were kept in a computer, producing the information in the plan descriptive (measures of centrality and dispersion) and, inferentially, Student $t$ test and One-way ANOVA. The main results show improvement in flexibility, explosive strength and speed and significant decrease in forward speed of 30 meters. KEY WORDS: Soccer; preparation; sports training; methodology. 
Evaluación del efecto del entrenamiento en el período preparatorio en atletas profesionales del fútbol.

RESUMEN: El objetivo del estudio fue evaluar los efectos de la formación en el período previo en el fútbol profesional. 17 atletas participantes, mayores de 18 años que compitió en la tercera división del Campeonato Paulista en 2009. El programa de formación fue el tipo predominante neuromuscular y de las pruebas de sentarse y llegar a, salto séxtuple, salto vertical y la velocidad de circulación en los $30 \mathrm{~m}$ atletas fueron evaluados antes de que el programa de capacitación ( $\mathrm{Ml}$ ) y después de siete semanas (M2). Los datos obtenidos fueron almacenados en un ordenador, la producción de información en el plan descriptivo (medidas de centralidad y dispersión) y, por inferencia, la prueba t de Student y ANOVA one way. .Los principales resultados muestran una mejora en la flexibilidad, fuerza explosiva y rápida y significativa disminución en la velocidad de avance de 30 metros.

PALABRAS CLAVE: Fútbol; preparación; entrenamiento deportivo; metodologia.

\section{REFERÊNCIAS}

BARROS, R. M. L. et al. Analysis of the distances covered by first division Brazilian soccer players obtained with an automatic tracking method. Journal of Sports Science and Medicine, v.6, p.233-242, 2007.

BEEDLE, B.; JESSE, C.; STONE, M. H. Flexibility characteristics among athletes who weight train. Journal of Applied Sport Science Research. v. 5, p. I50-54, 2001.

BELL, G. J. et al. Effect of concurrent strength and endurance training on skeletal muscle properties and hormone concentrations in humans. European Journal of Applied Physiology, v. 8I, p. 4I8-427, 2000.

BLOOMFIELD, J.; POLMAN, R.; O'DONOGHUE, P. Physical demands of different positions in FA Premier League soccer. Journal of Sports Sciences and Medicine, v.6, p.63-70, 2007.

BOGDANIS, G. et al. Effects of a hypertrophy and a maximal strength training program on speed, force and power of soccer players. Journal of Sports Science and Medicine, Suppl. I0, p.78-79, 2007.

BOSCO, C. A força muscular: aspectos fisiológicos e aplicações práticas. São Paulo: Phorte Editora, 2007.

BRAVO, D. F. et al. Sprint vs. Interval Training in Football. International Journal of Sports Medicine, v.29, p.668-674, 2008. 
BRAZ, T. V.; SPIGOLON, L. M. P.; BORIN, J. P. Proposta de bateria de testes para monitoramento das capacidades motoras em futebolistas. Revista da Educação Física/UEM. v. 20, n. 4, p. 569-575, 2009.

CASTAGNA, C. et al. Aerobic Fitness and Yo-Yo Continuous and intermittent tests performance in soccer players: a correlation study. Journal of Strength and Conditioning Research, Champaign, n.20, p.320-325, 2006.

COMETTI, G. et al. Isokinetic Strength and Anaerobic Power of Elite, Subelite and Amateur French Soccer Players. International Journal of Sports Medicine, Stuttgart, n.22, p.45-51, 200l.

CRONIN, J.; HANSEN, K. T. Resisted Sprint Training for the Acceleration Phase of Sprinting. Strength and Conditioning Journal, v.28, n.4, p.42-5I, 2006.

DI SALVO, V. et al. Performance Characteristics According to Playing Position in Elite Soccer. International Journal of Sports Medicine, v.28, n. 3, 2007.

FERRARI BRAVO, D. et al. Sprint vs. Interval training in football. International Journal of Sports Medicine, v.29, n. 17, p. 899-905, Dec, 2007.

FLECK, J. S.; KRAEMER, J. W. Fundamentos do Treinamento de Força Muscular. São Paulo: Artes Médicas Sul, 1999.

FORTEZA DE LA ROSA, A.; FARTO, E. R. Treinamento desportivo: do ortodoxo ao contemporâneo. São Paulo: Phorte, 2007.

GOMES, A. C.; SOUZA, J. Futebol: treinamento desportivo de alto rendimento. São Paulo: Editora Artmed, 2008.

GOROSTIAGA, E. M. et al. Effects of heavy resistance training on maximal and explosive force production, endurance and serum hormones in adolescent handball players. European Journal of Applied Physiology, v. 80, p.485-493, 1999.

GOROSTIAGA, E. M. et al. Strength training effects on physical performance and serum hormones in young soccer players. European Journal of Applied Physiology, v. 9I, p.698707, 2004.

HENNESSY, L. C.; WATSON, A. W. S. The interference effects of training for strength and endurance simultaneously. Journal of Strength and Conditioning Research, v. 8, p. I219, 1994.

HILL-HAAS, S. V. et al. Generic Versus Small-sided Game Training Soccer. International Journal of Sports Medicine, v.30, p.636-642, 2009.

HOFF, J. Training and testing physical capacities for elite soccer players. Journal of Sports Science, London, n. 23, p. 573-582, 2005. 
HOFF, J.; HELGERUD, J. Endurance and strength training for soccer players: physiological considerations. Sports Medicine, v. 34, p. I65-180, 2004.

IMPELLIZZERI, F. M. et al. Physiological and performance effects of generic versus specific aerobic training in soccer players. International Journal of Sports Medicine, v.27, p.483-492, 2006.

JONES, S.; BARRY, D. Physiological and technical demands of $4 \times 4$ and $8 \times 8$ games in elite youth soccer players. Kinesiology, v. 39, n. 2, p. 150- 156, 2007.

KELLY, D. M.; DRUST, B. The effect of pitch dimensions on heart rate responses and technical demands o small-sided soccer games in elite players. Journal of Science and Medicine in Sport, v. 12, n. 4, p. 475-479, Jul., 2008.

KOTZAMANIDIS, C. et al. The effect of a combined high-intensity strength and speed training program on the running and jumping ability of soccer players. Journal of Strength and Conditioning Research, v. 19, n. 2, p. 869-375, 2005.

KRAEMER, W. J. et al. Compatibility of high-intensity strength and endurance training on hormonal and skeletal muscle adaptations. Journal of Applied Physiology, v. 78, p. 976-989, 1995.

LEVERITT, M. et al. Concurrent strength and endurance training: a review. Sports Medicine, v. 28, p.413-427, 1999.

LITTLE, T.; WILLIAMS, A. G. Specificity of acceleration, maximum speed, and agility in professional soccer players. Journal of Strength and Conditioning Research, Champaign, n. 19 , p.76-78, 2005.

MARINS, J. C. B.; GIANNICHI, R. S. Avaliação e prescrição de atividade física: guia prático. Rio de Janeiro: Shape, 1996.

MCMILLAN, K. et al. Physiological adaptations to soccer specific endurance training in professional youth soccer players. British Journal of Sports Medicine, v.39, p.273-277, 2005.

NÚÑEZ, V. M. et al. Effects of training exercises for the development of strength and endurance in soccer. Journal of Strength and Conditioning Research, n.22, p.5 I 8-523, 2008.

PADOVANI, C. R. Noções Básicas de Estatística. In: CAMPANA, A. O.; PADOVANI, C. R.; IARIA, C. T. Investigação científica na área médica. São Paulo: Manole, 2001. p. 239-245.

PONTES, M. L. et al. Efeitos de 16 semanas de treinamento futebolístico na modalidade mobilidade dorso-lombar e isquiotibial de futebolistas amadores. In: CONGRESSO DE CIÊNCIA DO DESPORTO, I, 2005, Campinas. Anais... Campinas: FEF/NNICAMP, 2005, v. I, p. 28-32. 
RAMPININI, E. et al. Factors influencing physiological responses to small-sided soccer games. Journal of Sports Sciences, v.25, n.6, p.659-666, 2007.

REILLY, T. et al. A multidisciplinary approach to talent identification in soccer. Journal of Sports Science, London, n. 18, p. 695-702, 2000.

SAMPAIO, J. et al. Changes in speed, explosive strength and anaerobic power after application of two different training methods in soccer players. Journal of Sports Science and Medicine, Suppl. 10, p. I35-136, 2007.

SOUZA, E. N. Alterações das capacidades biomotoras de jovens futebolistas durante macrociclo de treinamento: a partir da periodização de cargas seletivas. 2006. $197 f$. Dissertação (Mestrado) - Curso de Mestrado em Educação Física, UNIMEP, Piracicaba, 2006.

STOLEN, T. et al. Physiology of soccer: an update. Sports Medicine, v. 35, p. 50 I-536, 2005.

SVENSSON, M.; DRUST, B. Testing soccer players. Journal of Sports Sciences. v. 23, n.6, p.60I-618, 2005.

TASKIN, H. Evaluating sprinting ability, density of acceleration, and speed dribbling ability of professional soccer players with respect to their positions. Journal of Strength and Conditioning Research, Champaign, n.22, p. |48|-|486, 2008.

THRASH, K.; KELLY, B. Flexibility and Strength training. Journal of Applied Sport Science Research, v. 4, p. 74-79, 2007.

TODD, T. Historical perspective: The myth of the muscle bound lifter. National Strength and Conditioning Association Journal, v. 7, p. 37-4I, 2005.

TOLEDO, N. Futebol: as cargas concentradas de força e dinâmica da alteração das capacidades bimotoras no Macrociclo anual de treinamento. 102 p. Dissertação (Mestrado) - Curso de Educação Física, Departamento de Ciências do Esporte, Universidade Estadual de Campinas, Campinas, 2000.

VENTURELLI, M.; TRENTIN, F; BUCCI, M. Strength training for young soccer players. Journal of Sports Science and Medicine, suppl. I0, p. 80-8I, 2007.

VERKHOSHANSKI, V. Y. (Org.) Hipertrofia Muscular - Body Building. Rio de Janeiro: 2000 .

WEINECK, E. J. Futebol Total: o treinamento físico no futebol. Guarulhos: Phorte, 2000.

WISLOFF, U. et al. Strong correlation of maximal squat strength with sprint performance and vertical jump height in elite soccer players. British Journal of Sports Medicine, n. 38 , p. 285-288, 2004. 
Recebido: 28 ago. 2009 Aprovado: 18 fev. 2010

Endereço para correspondência:

João Paulo Borin

Rua Armando Salles de Oliveira, 475

Bairro São Geraldo

Araraquara - SP

CEP: | 480|-335 